\title{
Neonatal brain microstructure correlates of neurodevelopment and gait in preterm children 18-22 mo of age: an MRI and DTI study
}

\author{
Jessica Rose ${ }^{1-3}$, Katelyn Cahill-Rowley ${ }^{1-4}$, Rachel Vassar ${ }^{1,2}$, Kristen W. Yeom ${ }^{5}$, Ximena Stecher ${ }^{6}$, David K. Stevenson ${ }^{7}$, \\ Susan R. Hintz ${ }^{7}$ and Naama Barnea-Goraly ${ }^{2}$
}

BACKGROUND: Near-term brain structure was examined in preterm infants in relation to neurodevelopment. We hypothesized that near-term macrostructural brain abnormalities identified using conventional magnetic resonance imaging (MRI), and white matter (WM) microstructure detected using diffusion tensor imaging (DTI), would correlate with lower cognitive and motor development and slower, less-stable gait at 18-22 mo of age.

METHODS: One hundred and two very-low-birth-weight preterm infants $(\leq 1,500 \mathrm{~g}$ birth weight; $\leq 32 \mathrm{wk}$ gestational age) were recruited prior to routine near-term brain MRI at $36.6 \pm 1.8$ wk postmenstrual age. Cerebellar and WM macrostructure was assessed on conventional structural MRI. DTI was obtained in 66 out of 102 and WM microstructure was assessed using fractional anisotropy and mean diffusivity (MD) in six subcortical brain regions defined by DiffeoMap neonatal atlas. Neurodevelopment was assessed with Bayley-Scales-of-InfantToddler-Development, 3rd-Edition (BSID-III); gait was assessed using an instrumented mat.

RESULTS: Neonates with cerebellar abnormalities identified using MRI demonstrated lower mean BSID-III cognitive composite scores ( $89.0 \pm 10.1$ vs. $97.8 \pm 12.4 ; P=0.002)$ at $18-22 \mathrm{mo}$. Neonates with higher DTI-derived left posterior limb of internal capsule (PLIC) MD demonstrated lower cognitive and motor composite scores $(r=-0.368 ; P=0.004 ; r=-0.354 ; P=0.006)$ at 18-22 mo; neonates with higher genu MD demonstrated slower gait velocity $(r=-0.374 ; P=0.007)$. Multivariate linear regression significantly predicted cognitive (adjusted $r^{2}=0.247$; $P=0.002$ ) and motor score (adjusted $r^{2}=0.131 ; P=0.017$ ).

CONCLUSION: Near-term cerebellar macrostructure and PLIC and genu microstructure were predictive of early neurodevelopment and gait.

A dvances in neonatal medicine have increased survival rates and improved outcome among very-low-birthweight (VLBW) preterm infants (1), but the incidence of neurodevelopmental impairment remains 3-4 times that of infants born full-term (2-5). Motor, cognitive, and behavioral impairments are difficult to predict accurately at an early age, limiting our ability to identify at-risk infants who could benefit from intervention during critical periods of development. Early identification at a time of optimal neuroplasticity is essential to guide treatment that has potential to improve function and quality of life for these children (6).

Neonatal neuroimaging data can help provide early prognosis for preterm infants, and most VLBW preterm infants undergo neuroimaging prior to discharge from the neonatal intensive care unit. Prognosis based on conventional brain magnetic resonance imaging (MRI) has been partially successful $(7,8)$. Semi-automated, atlas-based analysis of diffusion tensor imaging (DTI) is a promising neuroimaging technique that can be used to assess early white matter (WM) microstructure and may be a more sensitive biomarker of neurodevelopment than structural MRI (9-11).

As the brain develops, cellular microstructures become more complex and organized, water content decreases, and extracellular spaces diminish, changes that can be quantified with DTI $(12,13)$. Scalars obtained from DTI, such as fractional anisotropy (FA) and MD, can assess brain development and maturation (14). FA reflects degree of diffusion anisotropy within a voxel, determined by fiber diameter, density, myelination, extracellular diffusion, interaxonal spacing, and intravoxel fiber tract coherence. MD is a calculation of average diffusion along three main axes (15). In the neonate, WM FA generally increases and MD decreases with age, likely due to increased fiber organization, axonal coherence, and preliminary myelination $(11,16)$. Analysis of near-term regional WM development using DTI may provide prognostic information and help guide early treatment in children born preterm $(4,17)$.

In the neonate, rapidly developing WM is selectively vulnerable to injury (18) due to known risk factors associated with preterm birth, including bronchopulmonary dysplasia

'Department of Orthopaedic Surgery, Stanford University School of Medicine, Stanford, California; ${ }^{2}$ Neonatal Neuroimaging Laboratory, Stanford University School of Medicine, Stanford, California; ${ }^{3}$ Motion \& Gait Analysis Laboratory, Lucile Packard Children's Hospital, Stanford, California; ${ }^{4}$ Department of Bioengineering, Stanford University, Stanford, California; ${ }^{5}$ Department of Radiology, Lucile Packard Children's Hospital, Stanford University School of Medicine, Stanford, California; ${ }^{6}$ Department of Radiology, Universidad del Desarrollo, Desarrollo, Chile; ${ }^{7}$ Division of Neonatal and Developmental Medicine, Stanford University School of Medicine, Stanford, California. Correspondence: Jessica Rose (jessica.rose@stanford.edu) 
Table 1. Demographic and neonatal characteristics of all neonates and neonates with DTI

\begin{tabular}{|c|c|c|}
\hline & $\begin{array}{l}\text { All neonates } \\
\quad(n=102)\end{array}$ & $\begin{array}{l}\text { Neonates with } \\
\text { DTI }(n=66)\end{array}$ \\
\hline Males $n(\%)$ & $42(41)$ & $25(38)$ \\
\hline Females $n(\%)$ & $60(59)$ & $41(62)$ \\
\hline GA (weeks) mean (SD) & $28.7(2.4)$ & $28.9(2.3)$ \\
\hline BW (g) mean (SD) & $1,087(279)$ & $1,090(266)$ \\
\hline PMA at scan (weeks) mean (SD) $(n=102)$ & $36.6(1.8)$ & $36.5(1.3)$ \\
\hline Maternal age (years) mean (SD) $(n=99)$ & $31.6(6.0)$ & $31.9(6.1)$ \\
\hline Multiple gestation mean (SD) $(n=95)$ & $1.8(1.0)$ & $1.8(0.9)$ \\
\hline Apgar at 5-min mean (SD) $(n=100)$ & $7.4(1.9)$ & $7.5(1.7)$ \\
\hline Days on ventilation mean (SD) $(n=99)$ & $11.1(18.4)$ & $8.7(14.4)$ \\
\hline $\mathrm{BPD}^{\mathrm{a}}, n(\%)$ & $30(29)$ & $17(26)$ \\
\hline NEC, $n(\%)$ & $14(14)$ & $7(11)$ \\
\hline $\mathrm{ROP}^{\mathrm{b}}, n(\%)$ & $29(28)$ & $17(26)$ \\
\hline Sepsis ${ }^{c}, n(\%)$ & $12(12)$ & $6(9)$ \\
\hline Mean CRPd ${ }^{d}$ mg/dl, mean (SD) $(n=97)$ & $0.45(0.58)$ & $0.38(0.49)$ \\
\hline Peak CRPd, mg/dl, mean (SD) $(n=97)$ & $1.00(1.49)$ & $0.83(1.30)$ \\
\hline \multicolumn{3}{|c|}{$\begin{array}{l}\text { BPD, bronchopulmonary dysplasia; BW, birth weight; GA, gestational age; NEC, } \\
\text { necrotizing enterocolitis; PMA, postmenstrual age; ROP, retinopathy of prematurity; } \\
\text { aBPD defined as a history of respiratory distress syndrome, treated with oxygen }>21 \% \text { at } \\
36 \text { wk PMA. bPresence of ROP stage } 2 \text { or } 3 \text {. 'Sepsis, confirmed by positive blood culture. } \\
\text { dMeasured over first two postnatal weeks. }\end{array}$} \\
\hline
\end{tabular}

(BPD), necrotizing enterocolitis (NEC), retinopathy of prematurity (ROP), and sepsis (19-21). This study examined nearterm brain structure in VLBW preterm neonates in relation to neurodevelopment and gait at 18-22 mo of adjusted age. We hypothesized that near-term macrostructural brain abnormalities identified using conventional structural MRI and WM microstructure detected using DTI would correlate with lower cognitive and motor development and with slower, less stable gait at 18-22 mo of adjusted age.

\section{RESULTS}

Table 1 reports demographic and neonatal characteristics of all participants $(n=102)$ and of the subset population with DTI $(n=66)$. Gestational age (GA) at birth and birth weight were not significantly different in males and females. Demographic and clinical characteristics of infants with DTI $(n=66)$ and without DTI ( $n=38)$ were not significantly different; however, neonates with DTI had nonsignificant lower incidence of BPD (26 vs. $34 \%$ ), NEC (11 vs. $20 \%$ ), ROP (26 vs. $31 \%$ ), and sepsis ( 9 vs. 17\%); and nonsignificant fewer days on ventilation $(8.7 \pm 14.4$ vs. $14.4 \pm 23.2)$.

Representative images of WM abnormalities (WMA) and cerebellar abnormalities identified on conventional structural MRI are presented in Figure 1. We found that 92 (90\%) infants had no or mild WMA and 10 (10\%) had moderate-to-severe WMA. No WMA were found in 55 out of 102 (54\%), mild WMA in 37 out of 102 (36\%), moderate WMA in 8 out of 102 $(8 \%)$, and severe WMA in 2 out of $102(2 \%)$. Significant cerebellar abnormalities were found in 28 out of 102 (28\%), including

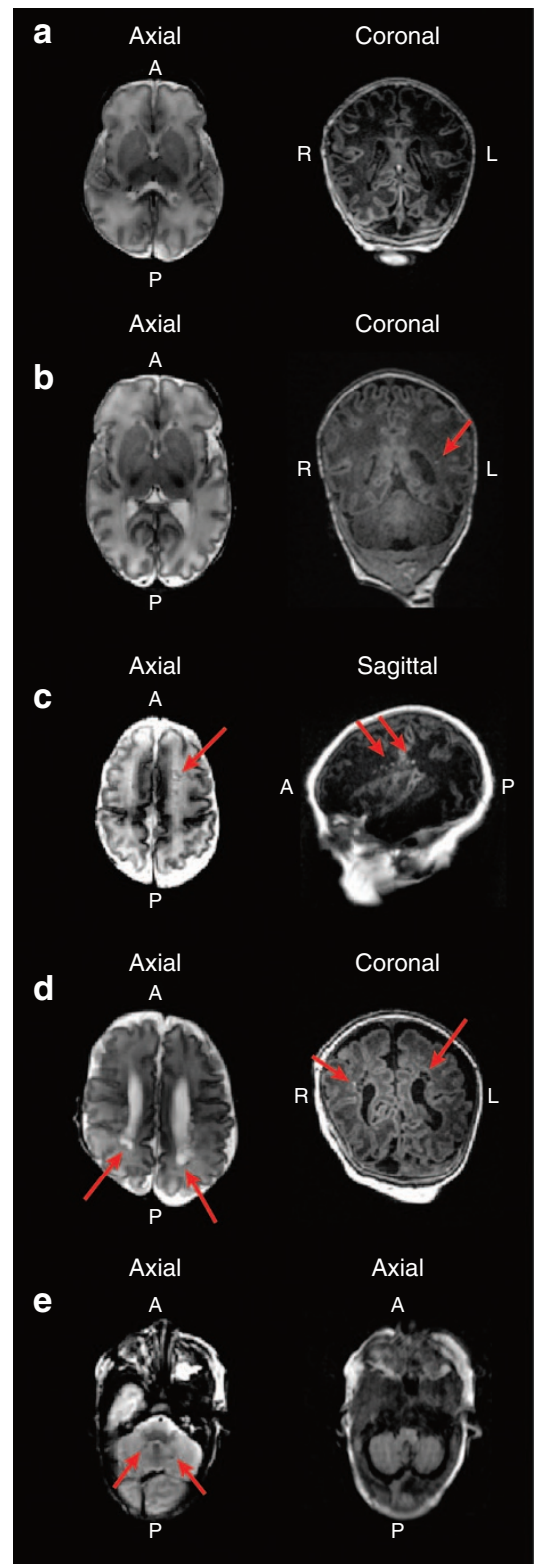

Figure 1. Macrostructural brain abnormalities assessed on representative $T_{1}$ and $\mathrm{T}_{2}$-weighted structural $\mathrm{MRI}(\mathrm{A}=$ anterior, $\mathrm{P}=$ posterior, $\mathrm{R}=$ right, $\mathrm{L}=$ left). (a) No WMA: note normal-appearing WM on both $\mathrm{T}_{2}$ and $\mathrm{T}_{1}$-weighted images without focal lesions or ventricular enlargement; (b) minimal WMA: note mild asymmetric ventricular enlargement of the left atrium on both $T_{2}$ - and $T_{1}$ weighted images, as well as focal $T_{1}$ high-intensity lesion (short-arrow) in the left periventricular WM; (c) moderate WMA: note focal cyst or cavity present in the left frontal WM (long arrow), as well as multiple $T_{1}$-high intensity focal lesions in the cerebral WM (short-arrows); (d) severe WMA: note multiple cysts or cavities located along periventricular WM (left image, long-arrows). Corresponding cystic/cavitary changes are again seen on T, SPGR volume sequence (right image, long-arrow), as well as more focal injury associated with $\mathrm{T} 1$ focal high intensity in the right periventricular WM (short-arrow). There is asymmetric mild ventricular dilatation, and deep extension of the cerebral sulci associated with overall cerebral WM volume loss; and (e) cerebellar abnormalities: multiple focal cerebellar lesions (short-arrows), likely representing combination of old blood products and/or injury. Note also asymmetric cerebellar volume, left smaller than right cerebellar hemisphere.

significant cerebellar lesions $(n=16)$ and/or significant asymmetry $(n=14), 73$ had no significant cerebellar abnormality, and for 1 participant it could not be conclusively determined. 
There were no significant differences between incidence of moderate-to-severe WMA or significant cerebellar abnormalities identified on MRI in males compared to females.

There were 6 out of 10 infants with moderate-to-severe WMA who also had cerebellar abnormalities. Five out of 10 infants with WMA had DTI; 15 out of 28 infants with cerebellar abnormalities had DTI.

Figure 2 shows selected brain regions assessed on nearterm DTI, as defined by the semi-automated Johns Hopkins University (JHU) neonatal atlas. Male sex was significantly associated with higher splenium FA $(P=0.012)$ and higher genu $\mathrm{MD}(P=0.006)$, after controlling for postmenstrual age (PMA) at scan; no other regions demonstrated significant associations. Presence of moderate-to-severe WMA on conventional MRI was significantly associated with higher genu MD $(P=0.008)$, after controlling for PMA at scan. Presence of cerebellar abnormalities on conventional MRI was significantly associated with higher left PLIC MD $(P=0.022)$ and higher thalamus MD $(P=0.049)$, after controlling for PMA at scan. No other significant associations were found.

Ninety-four out of 102 participants returned for follow-up evaluation at 18-22 mo of adjusted age; 93 children returned for Bayley-Scales-of-Infant-Toddler-Development, 3rd-Edition (BSID-III) evaluation ( $91 \%$ follow-up rate), 92 completed cognitive testing, 92 completed motor testing, and 91 completed both. There were three children who were diagnosed with cerebral palsy (CP) by 18-22 mo. Eighty-two children returned for gait assessment, which was not part of the standard-of-care

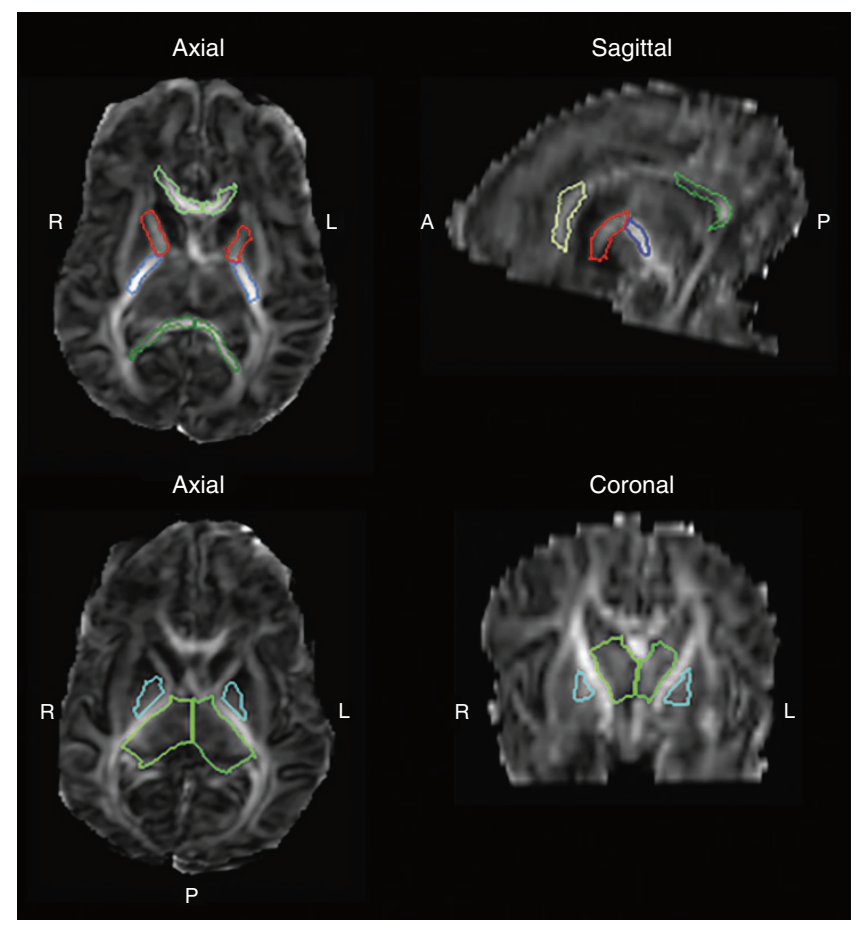

Figure 2. Brain white matter microstructure regions-of-interest selected on a representative infant DTI scan using the semi-automated JHU neonatal atlas ( $A=$ anterior, $P=$ posterior, $R=$ right, $L=$ left). Top images identify genu (light-green) and splenium (dark-green) of the corpus callosum and anterior (red) and posterior (blue) limbs of the internal capsule. Bottom images identify the thalamus (green) and globus pallidus (cyan). clinical follow-up; 78 out of 82 children walked independently, and 3 children were able to walk with handheld assist. One child was nonambulatory and unable to walk with handheldassist and was not included in gait analysis; a total of 81 participants completed gait assessment, mean age at follow-up was $20.1 \pm 1.0$ mo of adjusted age. None of the four children unable to walk independently had moderate-to-severe WMA, but two out of four children had significant cerebellar abnormalities, including the nonambulatory child. Among the 66 out of 102 participants who had near-term DTI, 60 children returned for BSID-III evaluation at 18-22 mo; 59 completed both cognitive and motor BSID-III testing; 52 completed gait evaluation.

Table 2 reports mean BSID-III scores and gait parameters at 18-22 mo for all children, for children with and without moderate-to-severe WMA, and for children with and without significant cerebellar abnormalities identified on near-term conventional MRI.

Mean BSID-III scores and gait parameters did not differ between males and females. There were 12 out of $92(13 \%)$ children who had BSID-III cognitive composite scores $<85$, and 15 out of 92 (16\%) had BSID-III motor composite scores $<85$, suggestive of mild-to-severe delay. There were 2 out of 92 (2\%) children who had cognitive scores $<70$ (scores of 65 and 65), suggestive of severe delay: one child had mild WMA and significant cerebellar abnormalities. The other child had no WMA or cerebellar abnormality, but GA at birth was $25 \mathrm{wk}$, and there was a history of ROP stage 3 , sepsis, and respiratory distress syndrome. There were 3 out of 92 (3\%) children who had motor scores $<70$ (scores of 49,58 , and 58), suggestive of severe delay: one child had severe WMA with no significant cerebellar abnormality, one child had mild WMA and significant cerebellar abnormality, and one child had no WMA but had significant cerebellar abnormality. Mean BSID-III scores and gait parameters were not significantly different in children with and without moderate-to-severe WMA. However, mean cognitive scores were significantly lower in children with cerebellar abnormalities ( $97.8 \pm 12.4$ vs. $89.0 \pm 10.1 ; P=0.002)$.

Table 3 reports mean DTI values and partial correlations between near-term DTI values and neurodevelopment and gait outcomes at 18-22 mo. Higher near-term left PLIC MD correlated with lower BSID-III composite cognitive and motor scores $(r=-0.368 ; P=0.004 ; r=-0.354 ; P=0.006$, respectively), with similar trends for right PLIC MD. Lower fine motor scores correlated with higher left and right PLIC $\operatorname{MD}(r=-0.368 ; P=0.004 ; r=-0.259 ; P=0.048$, respectively), higher thalamus $\mathrm{MD}$, and higher globus pallidus $\mathrm{MD}$, as well as lower PLIC FA and lower anterior limbs of the internal capsule (ALIC) FA (Table 3 ).

Univariate linear regression found that for each $1 \mathrm{SD}$ increase in left PLIC MD, BSID-III cognitive score was predicted to decrease by $0.354 \mathrm{SD}$ (standardized $B=0.354$; adjusted $\left.r^{2}=0.110 ; P=0.006\right)$ and BSID-III motor score was predicted to decrease by $0.358 \mathrm{SD}$ (standardized $B=0.358$; adjusted $\left.r^{2}=0.113 ; P=0.005\right)$.

Multivariate linear regression identified significant relationships between neurodevelopment at 18-22 mo adjusted age and 
Table 2. Mean BSID-III scores and gait parameters at 18-22 mo adjusted age for the total population, for those with and without moderate-tosevere WMA and for those with significant cerebellar abnormalities identified using near-term conventional structural MRI

\begin{tabular}{|c|c|c|c|c|c|c|c|}
\hline & $\begin{array}{l}\text { Cognitive } \\
\text { composite }\end{array}$ & $\begin{array}{l}\text { Motor } \\
\text { composite }\end{array}$ & $\begin{array}{l}\text { Fine } \\
\text { motor }\end{array}$ & $\begin{array}{l}\text { Gross } \\
\text { motor }\end{array}$ & $\begin{array}{l}\text { Gait velocity } \\
(\mathrm{cm} / \mathrm{s})\end{array}$ & $\begin{array}{c}\text { Gait SLS (L) } \\
\quad(\%)\end{array}$ & $\begin{array}{c}\text { Gait SLS (R) } \\
(\%)\end{array}$ \\
\hline $\begin{array}{l}\text { Mean (SD) } \\
\text { (range) }\end{array}$ & $\begin{array}{c}95.4(12.4) \\
(65-125) n=92\end{array}$ & $\begin{array}{c}93.9(12.6) \\
(49-127) n=92\end{array}$ & $\begin{array}{c}9.7(2.9) \\
(2-19) n=92\end{array}$ & $\begin{array}{c}8.3(2.3) \\
(1-12) n=92\end{array}$ & $\begin{array}{c}90.8(21.9) \\
(18-135) n=81\end{array}$ & $\begin{array}{c}40.3(4.1) \\
(20-49) n=81\end{array}$ & $\begin{array}{c}40.2(3.5) \\
(23-47) n=81\end{array}$ \\
\hline \multicolumn{8}{|l|}{ WMA } \\
\hline $\begin{array}{l}\text { Normal or mild } \\
\text { mean }(S D) ; n=92)\end{array}$ & $\begin{array}{c}95.6(12.2) \\
n=83\end{array}$ & $\begin{array}{c}93.9(11.8) \\
n=83\end{array}$ & $\begin{array}{c}9.7(2.9) \\
n=83\end{array}$ & $\begin{array}{c}8.4(2.2) \\
n=83\end{array}$ & $\begin{array}{c}90.7(22.4) \\
n=75\end{array}$ & $\begin{array}{c}40.4(4.2) \\
n=74\end{array}$ & $\begin{array}{c}40.2(3.7) \\
n=74\end{array}$ \\
\hline Significance $P=$ & 0.604 & 0.873 & 0.861 & 0.755 & 0.895 & 0.750 & 0.712 \\
\hline \multicolumn{8}{|l|}{ Cerebellar abnormality } \\
\hline $\begin{array}{l}\text { Normal or mild } \\
\text { mean (SD); } n=73\end{array}$ & $\begin{array}{c}97.8(12.4) \\
n=67\end{array}$ & $\begin{array}{c}94.5(12.7) \\
n=68\end{array}$ & $\begin{array}{c}9.9(3.0) \\
n=68\end{array}$ & $\begin{array}{c}8.4(2.2) \\
n=68\end{array}$ & $\begin{array}{c}91.9(22.1) \\
n=59\end{array}$ & $\begin{array}{c}40.4(3.9) \\
n=59\end{array}$ & $\begin{array}{c}40.5(3.7) \\
n=59\end{array}$ \\
\hline
\end{tabular}

BSID-III, Bayley-Scales-of-Infant-Toddler-Development, 3rd-Edition; MRI, magnetic resonance imaging; SLS, single limb support; WMA, white matter abnormalities.

${ }^{*} P<0.05$, two-tailed significance; ${ }^{* *} P<0.01$, two-tailed significance.

near-term MRI, DTI, and clinical risk factors. Specifically, multivariate analysis identified a model that included left PLIC MD controlled for PMA at scan, genu MD, cerebellar abnormality, average CRP, and sepsis significantly predicted BSID-III composite cognitive score (adjusted $r^{2}=0.247 ; d f=5 ; P=0.002$ ). Significant predictors in this model were left PLIC MD (standardized $B=-0.385 ; P=0.003$ ), and genu MD (standardized $B=0.318 ; P=0.013)$. Multivariate analysis identified that left PLIC MD, PMA at scan, and BPD significantly predicted BSIDIII composite motor score (adjusted $r^{2}=0.131 ; d f=2 ; P=0.007$ ). The significant predictor in this model was left PLIC MD (standardized $B=-0.358 ; P=0.005)$.

Slower gait velocity at $18-22$ mo of age correlated with higher near-term genu $\mathrm{MD}(r=-0.374 ; P=0.007)$ and lower genu FA $(r=0.322 ; P=0.021$; Table 3$)$. Univariate linear regression analysis found that for each 1SD increase in near-term genu MD, gait velocity was predicted to decrease by $0.376 \mathrm{SD}$ (standardized $B=0.376$, adjusted $r^{2}=0.124 ; P=0.006$; Figure 3 ). Shorter left and right single limb support (SLS) correlated to higher genu MD, and shorter left SLS correlated to lower genu FA (Table 3).

\section{DISCUSSION}

This study examined near-term brain structure on MRI and DTI in relation to neurodevelopment and gait at 18-22 mo. Results support the hypothesis and identify relationships that may have prognostic value for early identification of infants at risk for neurodevelopmental impairments.

\section{Near-Term Structural MRI and Neurodevelopment at 18-22 Mo}

Moderate-to-severe WMA at near-term age was not significantly associated with neurodevelopment or gait at 18-22 mo of age. However, the three children with BSID-III motor scores $<70$ had either moderate-to-severe WMA or significant cerebellar abnormality. Cerebellar abnormalities identified on near-term structural MRI were significantly associated with lower cognitive scores at 18-22 mo. Brain MRI scans commonly performed prior to discharge from the neonatal intensive care unit offer a potential opportunity to provide early prognosis; however, prognosis based on structural brain MRI has been only partially successful at detecting risk for motor deficits, cognitive delay, and language impairment later in life (22), consistent with our findings.

Evidence of moderate-to-severe WMA was identified in 10\% of infants, somewhat lower than two prior studies of extremely low birth weight (ELBW) infants, reporting an incidence of $17 \%$ (23) and 19\% (8). A lower occurrence of WMA may be expected among our study population, given their higher average GA. In addition, there may be a more uniform standardof-care delivered in the neonatal intensive care unit at this single-site study, compared to larger multisite studies (8).

In the preterm neonate, the rapidly developing cerebellum has been found to be a clinically important, vulnerable brain region (24-26), consistent with our findings. Evidence of significant cerebellar abnormalities was identified on MRI in $28 \%$ of infants; $16 \%$ had significant cerebellar lesions, an incidence somewhat higher than a prior report of $10 \%$ in ELBW preterm infants (8). Cerebellar abnormalities identified in this study included both significant cerebellar lesions as defined by Hintz et al. (8) and cerebellar asymmetry of $\geq 3 \mathrm{~mm}$, considered a clinically detectable degree of asymmetry, reflecting a volumetric difference, reproducibly identified and less prone to slice selection and infant positioning.

Comparison of conventional MRI and DTI findings revealed that moderate-to-severe WMA on MRI was associated with higher genu MD. Significant cerebellar abnormalities on MRI were associated with higher left PLIC MD and higher left thalamus MD. Further research may clarify relevant relationships between brain macrostructure and microstructure that can aid 
Articles | Roset al.

Table 3. Near-term regional DTI values in relation to BSID-III scores and gait parameters at 18-22 mo of age

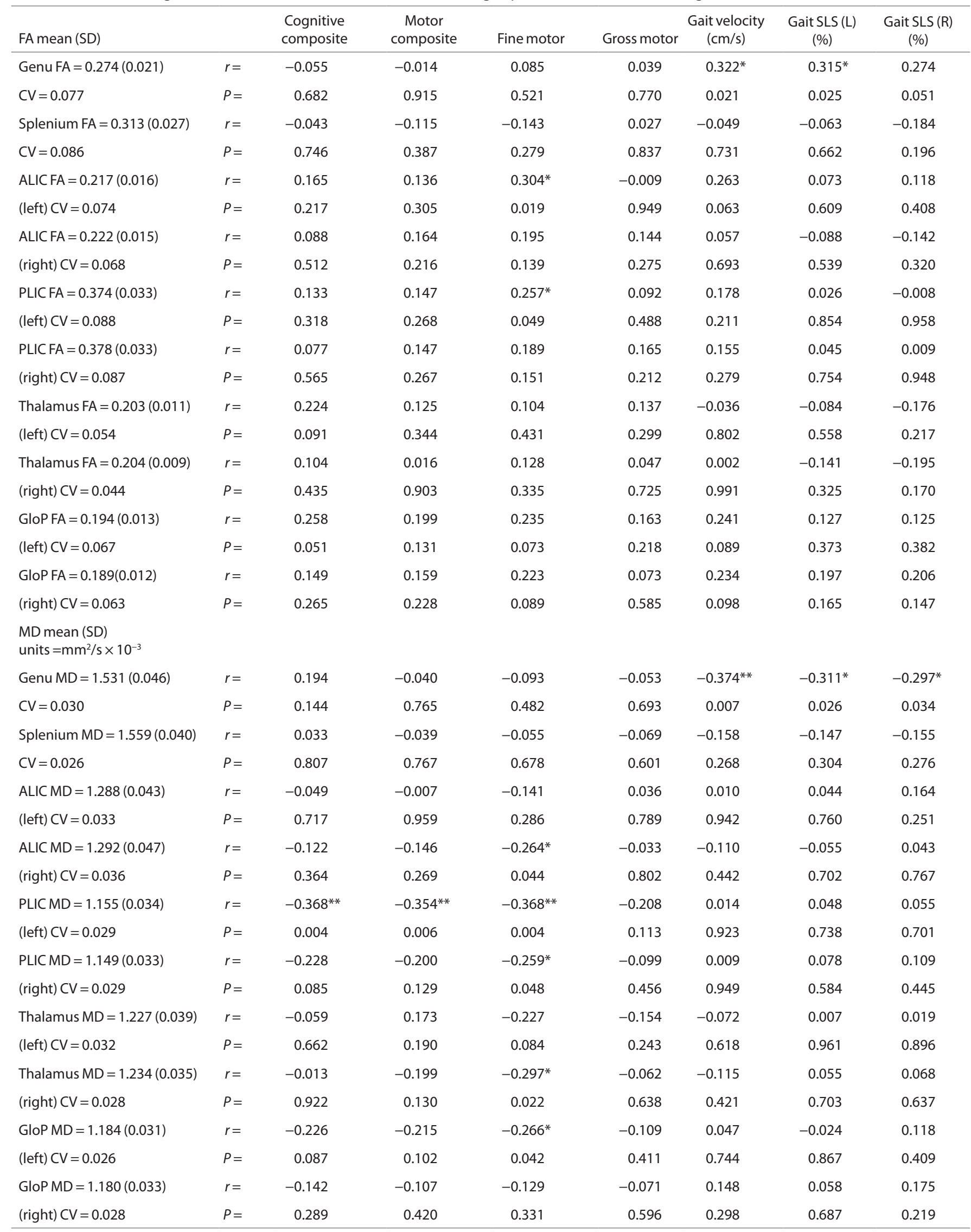

Partial correlation, corrected for PMA at scan $(r){ }^{*} P<0.05$, two-tailed significance; ${ }^{* *} P<0.01$, two-tailed significance. 
interpretation of conventional MRI, a more widely available method of neuroimaging.

\section{Near-Term DTI and Neurodevelopment at 18-22 Mo}

Near-term regional DTI, specifically, higher PLIC MD, correlated with lower BSID-III composite cognitive score, composite motor score, and fine motor score. Higher thalamus MD and higher GloP MD correlated with lower fine motor scores, as did lower ALIC FA. Multivariate analysis demonstrated that left PLIC MD was a significant predictor of cognitive and motor scores. Higher genu MD and lower FA correlated with slower, less stable gait.

While no significant differences in mean clinical characteristics were found between neonates with and without DTI, there were nonsignificant fewer days on ventilation and nonsignificant lower incidences of BPD, NEC, ROP, and sepsis which may indicate greater tolerance for the longer scan time required for DTI, collected at the end of the clinical scan, and suggests a need for shorter near-term scan time.

Prior studies suggested DTI as a more sensitive biomarker than structural MRI for later neurodevelopment $(4,11,27)$. Semi-automated, atlas-based DTI analysis has potential for clinical implementation because of its relative efficiency and repeatability. DTI was analyzed in WM regions known to mediate motor and cognitive function and reliably detected at near-term age by atlas-based DTI analysis (16). We previously reported WM development in 19 subcortical regions in a subpopulation of 45 out of 102 infants in this study (16). General patterns of central-to-peripheral regional WM development and posteriorto-anterior within-region WM development were observed. The PLIC demonstrated the highest level of WM development at near-term age. Results indicated that region-specific WM development trajectories are important to consider when interpreting DTI, consistent with prior findings, and near-term brain regions were selected for analysis, accordingly $(11,28)$.

We found that at near-term age, MD was more predictive of outcome than FA. MD and FA measure different histological characteristics. MD measures overall diffusion within a voxel, while FA measures degree of anisotropy, or directional water movement $(15,16)$. In the neonatal period, WM is expected to be less anisotropic as there is less myelin and more interstitial fluid and therefore MD may be a more sensitive measure. Additionally, MD values varied less than FA values. In this cohort, where a majority demonstrated normal neurodevelopment, a narrower variation may indicate a more sensitive metric.

\section{Corpus Callosum}

Lower FA and higher MD in the genu of the corpus callosum (CC) correlated with slower gait velocity and reduced SLS, a measure of gait stability. At near-term age, the genu and splenium of the CC have not yet myelinated (29), although posterior aspects (splenium) develop earlier than anterior (genu) aspects $(16,30)$. Lesion studies indicate that genu WM mediates motor coordination (31), and prior studies reported neonatal CC WM structure associated with future motor function $(32,33)$, consistent with our findings.

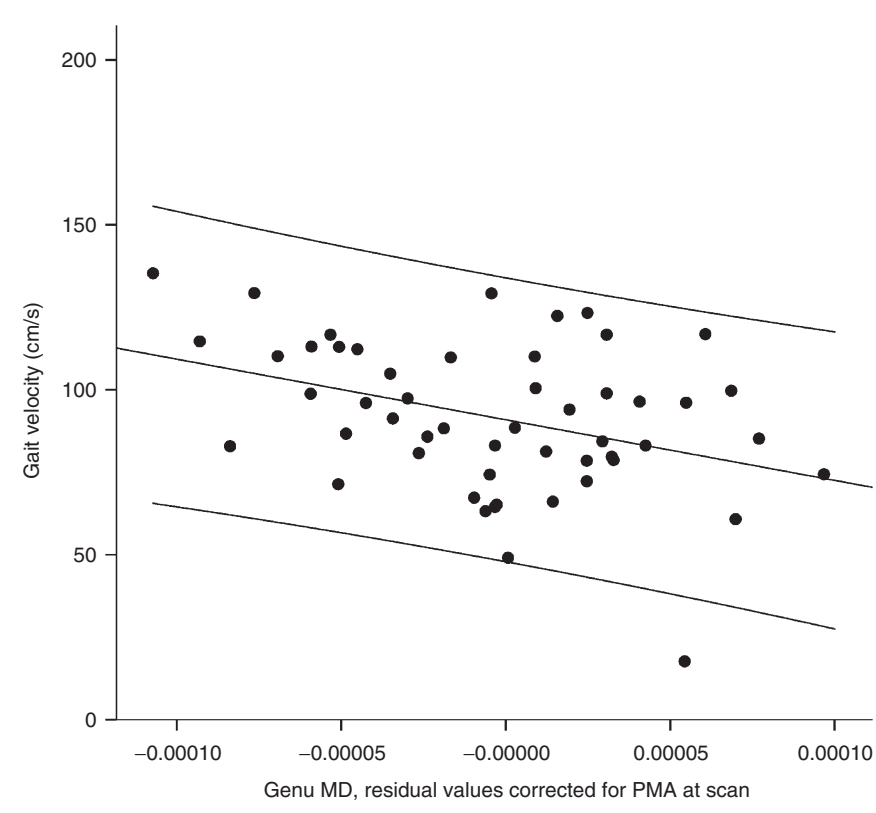

Figure 3. Gait velocity $(\mathrm{cm} / \mathrm{s})$ in VLBW preterm children at 18-22 mo of age in relation to near-term genu MD $(n=52)$, adjusted for PMA at scan (95\% confidence limits). Adjusted $r^{2}=0.124 ; P=0.006$.

Males had significantly higher splenium FA and higher genu MD than females, after controlling for PMA at scan. Limited data suggest quantifiable sex differences in CC development at near-term age $(4,34)$. Male gender is associated with increased risk of adverse outcomes, including $\mathrm{CP}$, following preterm birth (35), although males and females did not differ in neurodevelopmental outcome in this study. Associations between CC microstructure, sex, and neurodevelopment warrant further investigation in larger populations to explain risk factors that may increase morbidity among VLBW males.

\section{Internal Capsule}

Near-term PLIC microstructure demonstrated the strongest association with neurodevelopment at $18-22$ mo of age. Higher PLIC MD correlated with lower BSID-III cognitive composite score, motor composite score, and fine motor score, and lower PLIC FA was associated with lower fine motor score. The PLIC includes motor fibers of the corticospinal tract and is undergoing myelination during the near-term period (36). The corticospinal tract descends through the posterior third of the PLIC, and analyses of the PLIC on DTI at near-term age suggests that it may hold prognostic value for future motor development $(4,11)$.

In the late second and third trimester, developing WM is vulnerable to injury. WM injury may impair PLIC myelination and development through injury to pre-oligodendrocytes that fail to mature to myelin-producing oligodendrocytes, direct axonal injury, injury to transient neurons that facilitate cortical-subcortical synaptic connections, and injury to migrating neurons critical to cortical function $(7,18,37)$. Reduced PLIC maturation is likely reflected in lower PLIC FA and higher MD. Additionally, in the third trimester, microglia involved in normal apoptosis, vascularization, and axonal development reach peak abundance and 
are key effectors of cellular injury from ischemia or inflammation, further contributing to vulnerability (18).

The ALIC contains thalamocortical fibers, connecting the thalamus with prefrontal cortex to allow for motor planning and higher-order cognition (38). Near-term ALIC MD and FA values demonstrated correlations with BSID-III fine motor score, suggesting that microstructural injury to the ALIC may be associated with risk for fine motor impairment.

\section{Thalamus and Globus Pallidus}

Higher near-term thalamus and globus pallidus MD values correlated to lower BSID-III fine motor subscore at 18-22 mo of age, suggesting that less mature microstructure in these regions at near-term age may impact fine motor development in children born preterm. The thalamus and basal ganglia are known to mediate motor processes and have been found to be vulnerable to injury in infants $(7,39)$; which could partially explain impaired motor and cognitive function associated with preterm birth. In preterm compared to term infants, decreased thalamus volume (39) and impaired thalamocortical connectivity (40) have been reported. The thalamus undergoes significant development, establishing cortical connections during the late-second and third trimester of gestation (41), and this process may be disrupted by preterm birth.

Limitations of this study include relatively small sample size, cross-sectional neuroimaging analysis, the possibility that microscopic injury was below resolution of conventional MRI, DTI obtained in a subpopulation, and lack of information, such as maternal education, that may impact neurodevelopment. Future investigation within larger populations will further clarify relations identified in this study and may guide clinical implementation of near-term DTI to improve accuracy of early prognosis for preterm infants. Sequential neonatal neuroimaging may further reveal trajectories of brain development essential to neurodevelopment $(15,42)$.

In summary, these findings indicate that near-term cerebellar macrostructure and PLIC and genu microstructure may serve as prognostic biomarkers of cognitive and motor development, observations that can be further investigated in larger populations. Future studies may determine whether identification of these associations can provide accurate prognosis that may ultimately guide neuroprotective treatment and early intervention aimed at reducing neurodevelopmental disabilities in children born preterm.

\section{METHODS}

One hundred and two VLBW preterm infants were recruited, and consent was obtained from at least one parent or guardian for this Stanford University institutional review board-approved study, prior to routine near-term brain MRI conducted at $36.6 \pm 1.8 \mathrm{wk}$ PMA (1/2010-12/2011). The 102 participants represented $76 \%$ of eligible infants; 66 out of 102 also had successful DTI scans, collected at the end of standard-of-care MRI, before discharge from the neonatal intensive care unit (Table 1). VLBW preterm infants with no evidence of genetic disorders or congenital brain abnormalities were included.

\section{MRI Data Acquisition}

Brain MRI scans were performed on 3T MRI (GE-Discovery MR750, GE 8-Channel HD head coil, GE Healthcare, Little Chalfont, UK).
A three-plane localizer was used, and an asset calibration was prescribed to utilize parallel imaging. Sagittal $\mathrm{T}_{1}$ FLAIR image parameters were: $\mathrm{TE}=91.0, \mathrm{TR}=2,200$, field of view $(\mathrm{FOV})=20 \mathrm{~cm}$, matrix size $=320 \times 224$, slice thickness $3.0 \times 0.5 \mathrm{~mm}$ spacing, $\mathrm{NEX}=1 . \mathrm{T}_{2}$, DWI, and DTI axial scans were prescribed using a single acquisition, full-phase FOV. The axial fast spin echo $\mathrm{T}_{2}$ imaging parameters were: $\mathrm{TE}=85 \mathrm{~ms}, \mathrm{TR}=2,500, \mathrm{FOV}=20 \mathrm{~cm}$, matrix $=384 \times 224$; slice $=$ $4.0 \times 0.0 \mathrm{~mm}$ spacing. Axial T FLAIR parameters were: $\mathrm{TE}=140$, TR $=9,500, \mathrm{FOV}=20 \mathrm{~cm}$, slice $=4.0 \times 0.0 \mathrm{~mm}$, inversion time 2,300 fluid attenuated inversion recovery matrix $=384 \times 224$. Axial DWI parameters were: $\mathrm{TE}=88.8, \mathrm{TR}=10,000, \mathrm{FOV}=20 \mathrm{~cm}$, slice $=4.0 \times 0.0$ spacing, matrix $=128 \times 128$. Coronal $\mathrm{T}_{1} \mathrm{SPGR}$ parameters were: $\mathrm{TE}=$ $8, \mathrm{TR}=3$, slice $=1.0 \times 0.0$ spacing, $\mathrm{FOV}=24 \mathrm{~cm}$, matrix $=256 \times 256$.

The DTI scan was based on a diffusion-weighted, single-shot, spinecho, echo-planar imaging sequence with a slice thickness of $3.0 \mathrm{~mm}$, a matrix size of $128 \times 128$, a $90^{\circ}$ flip angle, $\mathrm{FOV}=20 \mathrm{~cm}, \mathrm{TE}=88.8 \mathrm{~ms}$, $\mathrm{TR}=8,000,\left(b=1,000 \mathrm{~s} / \mathrm{mm}^{2}\right)$. Diffusion was measured along 25 directions, with three $\mathrm{B} 0$ images. Two repetitions were obtained from 64 out of 66 subjects. MRI scans were performed as routine near-term neuroimaging for preterm infants, and the DTI sequences were collected at the end of these 25 -min MRI scans. Infants were swaddled and fed and typically remained asleep during the scan. Sedation was not utilized as part of the research protocol and was typically not utilized for routine near-term MRI.

\section{Radiological Assessment}

Structural MRI was assessed for degree of WMA and significant cerebellar abnormality. Radiological evaluation was performed by an experienced pediatric neuroradiologist (X.S.) and confirmed by a second (K.Y.), both were masked to all other participant data. A form validated for near-term neuroradiological assessment (8) was used to score WMA (1-3) according to a widely used classification system $(8,23,43)$ : (i) extent of WM signal abnormality, (ii) periventricular WM volume loss, (iii) cystic abnormalities, (iv) ventricular dilation, and (v) thinning of the CC. High inter-rater agreement (96-98\%) for moderate-tosevere WMA using this classification was reported $(8,43)$. Significant cerebellar abnormalities included significant cerebellar lesions defined by Hintz et al. (8) and/or significant cerebellar asymmetry of $\geq 3 \mathrm{~mm}$ in the anterior-posterior or medial-lateral direction.

\section{DTI Processing}

DTI was collected at the end of the scan, and in 31 out of 102 infants, the scan was terminated prior to collecting DTI, primarily due to the infant waking up, 2 did not receive DTI due to technical problems, DTI collected from 3 infants was discarded due to head movement and poor quality. In a subpopulation of 66 infants, semi-automated, atlas-based DTI was analyzed using DTI-Studio using parameters outlined in Oishi et al. (44). A trained investigator inspected all scans; 64 out of 66 infants had two repetitions, and the best repetition was selected to eliminate images with artifacts or evidence of motion. If no full repetition was usable (2/66), a composite repetition was generated based on best image slices. Automated Image Registration was performed using an affine transformation to further correct for eddy current distortions and minor head motion.

Each brain image was skull-stripped with ROI Editor using B0 and trace images and manually rotated to align with the JHU neonatal template image. DTI images were processed with DiffeoMap (MRIStudio, Baltimore, MD,) using FA and trace (the sum of the three orthogonal diffusivities per voxel) maps to perform a large deformation diffeomorphic metric mapping transformation (44). FA and trace maps from each brain were normalized to map onto the neonatal atlas and segmented into 126 regions. Based on the trace map, regions $>0.006 \mathrm{~mm}^{2} / \mathrm{s}$ were considered CSF and excluded from the segmented regions. A threshold of FA $>0.15$ was then applied to segment developing WM tracts (30). Regions selected were then mapped onto the FA and trace maps, and regional values were obtained. MD was defined as an average of the three directions of diffusion calculated from the trace image. We examined subcortical WM regions defined by JHU parcellation atlas that were well visualized with near-term MRI and DTI, and known to mediate motor and cognitive function (Figure 2). 


\section{Demographic and Neonatal Characteristics}

Clinical risk factors included: BPD, defined as a history of respiratory distress syndrome and treated with oxygen $>21 \%$ at 36 wk PMA; sepsis confirmed with positive blood culture; presence of NEC; and presence of stage 2 or 3 ROP. Serum CRP was sampled $3.72 \pm 1.9$ times over the first 2 postnatal wk, obtained through a search conducted with Stanford Center for Clinical Informatics and STRIDE. Analysis of neonatal clinical risk factors and neuroimaging in the same cohort was previously published (21).

\section{Neurodevelopmental Assessment at 18-22 Mo}

Neurodevelopment was assessed using the BSID-III at 18-22 mo of age, adjusted for prematurity, conducted by a trained examiner masked to clinical data and neuroimaging. Composite cognitive and motor scores, and fine and gross motor subscores were calculated based on the child's adjusted age at the time of evaluation. The BSID-III was conducted in English or Spanish based on the child's primary language.

Gait velocity and SLS, calculated as a percent of the gait cycle, were assessed at 18-22 mo adjusted age using a GAITRite instrumented mat (CIR Systems, Sparta, NJ). Walking trials met three criteria: (i) at least four consecutive footfalls, (ii) at least one foot on the ground at any given time, and (iii) the child did not touch or carry anything. If a child was not walking independently at follow-up, this was noted, and data was included if they were able to cruise and could walk with handheld-assist. For each child, 2-3 "best effort" trials with a total of at least 12 footfalls were analyzed, selected based on walk length, straightness, and pace consistency.

\section{Statistical Analysis}

Associations between categorical variables were analyzed using Chisquared tests, $\alpha=0.05$. Mean differences were compared using $t$-test, and two-tailed significance $P<0.05$. Mean DTI values were compared in males and females, and in infants with and without WMA and cerebellar abnormalities using analysis of covariance, with PMA at scan as a covariate, and significance $P<0.05$. Partial correlations for DTI, controlled for PMA at scan, with significance $P<0.05$. To evaluate slope of the relationship between DTI and outcome, univariate linear regression was performed with residual MD values, correcting for PMA at scan. Multivariate linear regression was performed for candidate variables that had correlations with outcome $(P<0.20)$. Correlations among candidate variables were calculated and if variables correlated $>0.6$, the variable with highest correlation to outcome was selected. This analysis aimed to identify promising relations for future studies; therefore, an alpha of 0.05 was selected to protect against type-II error; exact $P$ values are provided so it can be determined which results would remain, had a lower alpha been selected.

\section{ACKNOWLEDGMENTS}

We thank Alex Harris, John Tamaresis, Ron Cohen, Allan White, Elizabeth Loi, Megan Thompson, Anne DeBattista, Anne Fernald, and Heidi Feldman for valuable consultation and discussions.

\section{STATEMENT OF FINANCIAL SUPPORT}

This research is supported by the National Institutes of Health, Bethesda MD, Clinical and Translational Science Award UL1 RR025744 for the Stanford Center for Clinical and Translational Education and Research (Spectrum) and for the Stanford Center for Clinical Informatics and Stanford Translational Research Integrated Database Environment (STRIDE), Stanford, CA; and by the Lucile Packard Foundation for Children's Health, Palo Alto, CA. This material is based on work supported by the National Science Foundation, Arlington, VA, Graduate Research Fellowship under Grant No. DGE-1147470, and was supported by the Mary Baracchi Research Fund, Lucile Packard Children's Hospital, Stanford.

Disclosure: The authors have stated that they had no interest that might be perceived as posing a conflict or bias, and there was no involvement of funders in study design, data collections, data analysis, manuscript preparation, and/or publication decisions.

\section{REFERENCES}

1. Moore T, Hennessy EM, Myles J, et al. Neurological and developmental outcome in extremely preterm children born in England in 1995 and 2006: the EPICure studies. BMJ 2012;345:e7961.

2. Spittle AJ, Cheong J, Doyle LW, et al. Neonatal white matter abnormality predicts childhood motor impairment in very preterm children. Dev Med Child Neurol 2011;53:1000-6.

3. Litt J, Taylor HG, Klein N, Hack M. Learning disabilities in children with very low birthweight: prevalence, neuropsychological correlates, and educational interventions. J Learn Disabil 2005;38:130-41.

4. Rose J, Butler EE, Lamont LE, Barnes PD, Atlas SW, Stevenson DK. Neonatal brain structure on MRI and diffusion tensor imaging, sex, and neurodevelopment in very-low-birthweight preterm children. Dev Med Child Neurol 2009;51:526-35.

5. Woodward LJ, Clark CA, Bora S, Inder TE. Neonatal white matter abnormalities an important predictor of neurocognitive outcome for very preterm children. PLoS One 2012;7:e51879.

6. Maulik PK, Darmstadt GL. Community-based interventions to optimize early childhood development in low resource settings. J Perinatol 2009;29:531-42.

7. Miller SP, Ferriero DM. From selective vulnerability to connectivity: insights from newborn brain imaging. Trends Neurosci 2009;32:496-505.

8. Hintz SR, Barnes PD, Bulas D, et al.; SUPPORT Study Group of the Eunice Kennedy Shriver National Institute of Child Health and Human Development Neonatal Research Network. Neuroimaging and neurodevelopmental outcome in extremely preterm infants. Pediatrics 2015;135:e32-42.

9. Arzoumanian Y, Mirmiran M, Barnes PD, et al. Diffusion tensor brain imaging findings at term-equivalent age may predict neurologic abnormalities in low birth weight preterm infants. AJNR Am J Neuroradiol 2003;24:1646-53.

10. Rose J, Mirmiran M, Butler EE, et al. Neonatal microstructural development of the internal capsule on diffusion tensor imaging correlates with severity of gait and motor deficits. Dev Med Child Neurol 2007;49: 745-50.

11. De Bruïne FT, Van Wezel-Meijler G, Leijser LM, et al. Tractography of white-matter tracts in very preterm infants: a 2-year follow-up study. Dev Med Child Neurol 2013;55:427-33.

12. Dubois J, Dehaene-Lambertz G, Perrin M, et al. Asynchrony of the early maturation of white matter bundles in healthy infants: quantitative landmarks revealed noninvasively by diffusion tensor imaging. Hum Brain Mapp 2008;29:14-27.

13. Oishi K, Faria AV, Yoshida S, Chang L, Mori S. Quantitative evaluation of brain development using anatomical MRI and diffusion tensor imaging. Int J Dev Neurosci 2013;31:512-24.

14. Pierpaoli C, Jezzard P, Basser PJ, Barnett A, Di Chiro G. Diffusion tensor MR imaging of the human brain. Radiology 1996;201:637-48.

15. Chen $\mathrm{Y}, \mathrm{An} \mathrm{H}, \mathrm{Zhu} \mathrm{H}$, et al. Longitudinal regression analysis of spatialtemporal growth patterns of geometrical diffusion measures in early postnatal brain development with diffusion tensor imaging. Neuroimage 2011;58:993-1005.

16. Rose J, Vassar R, Cahill-Rowley K, Guzman XS, Stevenson DK, BarneaGoraly N. Brain microstructural development at near-term age in verylow-birth-weight preterm infants: an atlas-based diffusion imaging study. Neuroimage 2014;86:244-56.

17. Hüppi PS, Dubois J. Diffusion tensor imaging of brain development. Semin Fetal Neonatal Med 2006;11:489-97.

18. Volpe JJ. The encephalopathy of prematurity-brain injury and impaired brain development inextricably intertwined. Semin Pediatr Neurol 2009;16:167-78

19. van Vliet EO, de Kieviet JF, Oosterlaan J, van Elburg RM. Perinatal infections and neurodevelopmental outcome in very preterm and very lowbirth-weight infants: a meta-analysis. JAMA Pediatr 2013;167:662-8.

20. Mitha A, Foix-L'Hélias L, Arnaud C, et al.; EPIPAGE Study Group. Neonatal infection and 5-year neurodevelopmental outcome of very preterm infants. Pediatrics 2013;132:e372-80. 


\section{Articles | Rose et al.}

21. Rose J, Vassar R, Cahill-Rowley K, et al. Neonatal physiological correlates of near-term brain development on MRI and DTI in very-low-birthweight preterm infants. Neuroimage Clin 2014;5:169-77.

22. Kidokoro H, Anderson PJ, Doyle LW, Neil JJ, Inder TE. High signal intensity on T2-weighted MR imaging at term-equivalent age in preterm infants does not predict 2-year neurodevelopmental outcomes. AJNR Am J Neuroradiol 2011;32:2005-10.

23. Horsch S, Skiöld B, Hallberg B, et al. Cranial ultrasound and MRI at term age in extremely preterm infants. Arch Dis Child Fetal Neonatal Ed 2010;95:F310-4.

24. Volpe JJ. Cerebellum of the premature infant: rapidly developing, vulnerable, clinically important. J Child Neurol 2009;24:1085-104.

25. Limperopoulos C, Chilingaryan G, Sullivan N, Guizard N, Robertson RL, du Plessis AJ. Injury to the premature cerebellum: outcome is related to remote cortical development. Cereb Cortex 2014;24: $728-36$.

26. Van Kooij BJ, Benders MJ, Anbeek P, Van Haastert IC, De Vries LS, Groenendaal F. Cerebellar volume and proton magnetic resonance spectroscopy at term, and neurodevelopment at 2 years of age in preterm infants. Dev Med Child Neurol 2012;54:260-6.

27. Goergen SK, Ang H, Wong F, et al. Early MRI in term infants with perinatal hypoxic-ischaemic brain injury: interobserver agreement and MRI predictors of outcome at 2 years. Clin Radiol 2014;69:72-81.

28. Kersbergen KJ, Leemans A, Groenendaal F, et al. Microstructural brain development between 30 and 40 weeks corrected age in a longitudinal cohort of extremely preterm infants. Neuroimage 2014;103:214-24.

29. Provenzale JM, Isaacson J, Chen S. Progression of corpus callosum diffusion-tensor imaging values during a period of signal changes consistent with myelination. AJR Am J Roentgenol 2012;198:1403-8.

30. Vassar RL, Barnea-Goraly N, Rose J. Identification of neonatal white matter on DTI: influence of more inclusive thresholds for atlas segmentation. PLoS One 2014;9:e115426.

31. Caillé S, Sauerwein HC, Schiavetto A, Villemure JG, Lassonde M. Sensory and motor interhemispheric integration after section of different portions of the anterior corpus callosum in nonepileptic patients. Neurosurgery 2005;57:50-9; discussion 50-9.
32. Counsell SJ, Edwards AD, Chew AT, et al. Specific relations between neurodevelopmental abilities and white matter microstructure in children born preterm. Brain 2008;131(Pt 12):3201-8.

33. Thompson DK, Inder TE, Faggian N, et al. Corpus callosum alterations in very preterm infants: perinatal correlates and 2 year neurodevelopmental outcomes. Neuroimage 2012;59:3571-81.

34. van Kooij BJ, van Pul C, Benders MJ, van Haastert IC, de Vries LS, Groenendaal F. Fiber tracking at term displays gender differences regarding cognitive and motor outcome at 2 years of age in preterm infants. Pediatr Res 2011;70:626-32.

35. Beaino G, Khoshnood B, Kaminski M, et al.; EPIPAGE Study Group. Predictors of cerebral palsy in very preterm infants: the EPIPAGE prospective population-based cohort study. Dev Med Child Neurol 2010;52:e119-25.

36. Yakovlev PI, Lecours A. The myelogenetic cycles of regional maturation of the brain. In: Minkowski A, ed. Regional Development of the Brain in Early Life. Oxford: Blackwell, 1967:3-70.

37. Liu XB, Shen Y, Plane JM, Deng W. Vulnerability of premyelinating oligodendrocytes to white-matter damage in neonatal brain injury. Neurosci Bull 2013;29:229-38.

38. Nolte J. The Human Brain: An Introduction to Its Functional Anatomy. 6th edn. Philadelphia, PA: Mosby/Elsevier, 2009.

39. Ball G, Boardman JP, Rueckert D, et al. The effect of preterm birth on thalamic and cortical development. Cereb Cortex 2012;22:1016-24.

40. Ball G, Boardman JP, Aljabar P, et al. The influence of preterm birth on the developing thalamocortical connectome. Cortex 2013;49:1711-21.

41. Kostović I, Judas M. The development of the subplate and thalamocortical connections in the human foetal brain. Acta Paediatr 2010;99:1119-27.

42. Chau V, Synnes A, Grunau RE, Poskitt KJ, Brant R, Miller SP. Abnormal brain maturation in preterm neonates associated with adverse developmental outcomes. Neurology 2013;81:2082-9.

43. Woodward LJ, Anderson PJ, Austin NC, Howard K, Inder TE. Neonatal MRI to predict neurodevelopmental outcomes in preterm infants. $\mathrm{N}$ Engl J Med 2006;355:685-94.

44. Oishi K, Mori S, Donohue PK, et al. Multi-contrast human neonatal brain atlas: application to normal neonate development analysis. Neuroimage 2011;56:8-20. 